\title{
Endovascular Treatment for Trans-Atlantic Inter-Society Consensus II D Femoropopliteal Arterial Disease in Elderly Patients with Ulcer and Gangrene: A Retrospective Analysis
}

\author{
Peng Liu ${ }^{1}$, Li-na Yan ${ }^{2}$, Hai-xia Song ${ }^{3}$, Zi-bin Wang ${ }^{1}$, Miao Zhang ${ }^{1}$, Xu-peng Niu ${ }^{1}$, Xin-qi He ${ }^{1}$, and Zhen-yu $\mathrm{Wu}^{1}{ }^{1, *}$ \\ ${ }^{1}$ Department of General Surgery, First Hospital of Hebei Medical University, Shijiazhuang, Hebei 050031, China \\ ${ }^{2}$ Department of Medical Insurance, The First Hospital of Hebei Medical University, Shijiazhuang, Hebei 050031, China \\ ${ }^{3}$ Department of Neurology, The People's Hospital of Shijiazhuang, Shijiazhuang, Hebei 050011, China
}

*Corresponding author: Zhen-yu Wu, Department of General Surgery, The First Hospital of Hebei Medical University, No. 89, Dong gang Road, Shijiazhuang, Hebei 050031, China, E-mail: 254657192@qq.com

Received: 28 Apr, 2021 | Accepted: 25 May, 2021 | Published: 01 Jun, 2021

Citation: Liu P, Yan LN, Song HX, Wang ZB, Zhang M, et al. (2021) Endovascular Treatment for Trans-Atlantic Inter-Society Consensus II D Femoropopliteal Arterial Disease in Elderly Patients with Ulcer and Gangrene: A Retrospective Analysis. J Surg Open Access 7(5): dx.doi. org/10.16966/2470-0991.240

Copyright: (C2021 Liu P, et al. This is an open-access article distributed under the terms of the Creative Commons Attribution License, which permits unrestricted use, distribution, and reproduction in any medium, provided the original author and source are credited.

\begin{abstract}
Objective: The aim of this study was to analyze the middle-term results of endovascular treatment for Trans-Atlantic Inter-Society (TASC) II D femoropopliteal arterial disease in elderly patients with ulcer and gangrene.

Methods: The data of 39 elderly patients with TASCII D femoropopliteal artery disease who underwent endovascular treatment at the First Hospital of Hebei Medical University were retrospectively analyzed. The clinical characteristics, vascular patency/opening) rate, survival rate, limb salvage rate, ulcer healing rate were studied.

Results: The mean age of 39 patients was $70.5 \pm 6.4$ years. 25 cases of ulcers and 14 cases of gangrene were all treated by endovascular treatment. The mean lesion length was $32.0 \pm 7.1 \mathrm{~cm}$. The postoperative Ankle-Brachial Index (ABI) was significantly higher than that of the preoperative ABI $(\mathrm{P}<0.05)$. The technical and clinical success rate was $100 \%(39 / 39)$, the perioperative cardio-cerebrovascular event rate was $7.7 \%(3 / 39)$, and the in-stent restenosis or occlusion was 43.6\% (17/39). Primary patency rate at 1 year, 2 years and 3 years were $94.5 \%, 75.1 \%$, and $55.6 \%$, respectively. Overall survival rates at 1 year, 2 years and 3 years were $89.7 \%, 76.9 \%$, and $67.3 \%$, respectively. Limb salvage rates at 1 year, 2 years and 3 years were $71.8 \%, 64.1 \%$ and $53.5 \%$, respectively. On univariate analysis, risk factors affecting primary patency included common femoral artery lesions, diabetes, and end stage renal disease. Risk factors affecting the overall survival rate included end stage renal disease, coronary heart disease and gangrene. Risk factors affecting the limb salvage rate were age, diabetes, end stage renal disease, and gangrene. The ulcer healing rate was $88.0 \%$ $(22 / 25)$, and the mean healing time was $38.3 \pm 17.2$ days.
\end{abstract}

Conclusions: Endovascular treatment for TASC II D femoropopliteal artery disease in elderly patients with ulcer and gangrene was safe and effective, and worthy of clinical application.

Keywords: Periphery artery disease; Ulcer and gangrene; Elderly patient; Patency rate; Survival; Limb salvage

\section{Introduction}

Peripheral Artery Disease (PAD) is a common circulatory problem, caused by narrowed arteries, resulting in atherosclerotic obstructions in the major conduit arteries [1]. PAD covers different stages from asymptomatic stenosis to ischemic amputation [2]. Ulcer gangrene, as the main manifestation of severe ischemia, seriously affects the quality of life of elderly patients and increases the social and economic burden [3]. The incidence rate of PAD is about $3 \%-10 \%$ and can increase to $15 \%-20 \%$ in the elder people over 70 years [4]. Severe PAD cases with ulcers or gangrene have to be amputated or even died. Surgery is the first choice for severe PAD patients. For the choice of treatment strategy, patients with life expectancy $>2$ years underwent open surgery, while patients with life expectancy $<2$ years received endovascular treatment [5]. In recent years, with the accumulation of clinicians' experience and the development of technical materials, endovascular treatment of complex long segment lesions of lower limbs has become a common phenomenon [6].

Based on the evidence-based medical evidence, Trans-Atlantic Inter-Society Consensus II (TASCII) re-classified the femoral popliteal artery lesions and defines class $\mathrm{D}$ femoropoliteal lesions as chronic total occlusion of $>20 \mathrm{~cm}$ of the Common Femoral Artery (CFA) or Superficial Femoral Artery (SFA) involving the popliteal artery and chronic total occlusion of the popliteal artery and chronic total occlusion of the popliteal artery involving proximal trifurcation 
vessels suggested open surgery for TASC II D femoropopliteal arterial disease [4,5]. In addition, with the development of interventional material technology in recent years, endovascular therapies has been widely used in the operation of TASCII D lesions, and have achieved good clinical results [7-9]. In this study, we analyzed the clinical data of elderly patients with TASC II D femoropopliteal arterial disease, who received endovascular treatment in the first hospital of Hebei Medical University, and discussed its curative effect.

\section{Patients and Methods}

\section{Patients}

From February 2014 to February 2018, 39 patients with TASC II D femoral popliteal artery occlusion received endovascular treatment in the first hospital of Hebei Medical University. The patients included 29 males and 10 females, aged from 60 to 82 years. 27 were for Common Femoral Artery (CFA) occlusion of $>20 \mathrm{~cm}$ in length and 12 were for popliteal artery occlusions involving the trifurcation vessels. Ankle Brachial Index (ABI), color Doppler ultrasound and arterial imaging were performed in all patients.

Clinical diagnosis of PAD refers to the guideline [4], and the severity of the diseases was based on fontaine stages. The inclusion criteria were as following: age $>60$ years; chronic total occlusion length of common femoral artery and superficial femoral artery $>20 \mathrm{~cm}$, involving popliteal artery [5] at least one inferior genicular artery was unobstructed (stenosis rate $<30 \%$ ), proximal end of occluded segment was unobstructed (stenosis rate $<30 \%$ ). Exclusion criteria were as following: contrast media allergy, antiplatelet and anticoagulant contraindications, previous interventional therapy of target vessels, arterial thrombosis, and thromboangiitis obliterans. The indication of stent implantation was residual stenosis $>50 \%$ or limited dissection. Technical success was defined as residual stenosis $<30 \%$ and positive blood flow. Clinical success was defined as remission of ischemic symptoms and increase of ABI. Primary patency was defined as the patency of the target vessel without reoperation. Auxiliary primary patency was defined as the patency of blood flow after intracavitary treatment for diseased vessels other than target vessels. The secondary patency was defined as the occlusion of the target vessel after successful primary treatment and patency after interventional therapy [10]. Overweight and obesity were defined as Body Mass Index (BMI) $>24.0$. The area method was used to calculate the size of ulcer. The healing time was calculated from the operation date to the last followup or ulcer healing day [11]. Major amputation was above the level of ankle joint. The small amputation was amputated at and below the ankle joint. The follow-up included the recurrence and aggravation of ischemic symptoms, vascular stenosis and occlusion, health education, etc.

\section{Anesthesia operation}

All the affected limbs received endovascular treatment. Local anesthesia was the first choice, followed by general anesthesia. The femoral artery of the affected side or the uninjured side was selected for antegrade or reverse puncture. Before operation, heparinization (intravenous injection of unfractionated $100 \mathrm{IU} / \mathrm{kg}$ heparin) was performed. The target vessel was opened in the true lumen first, and the subintimal opening technique was used after failure. If the opening failed, the reverse puncture technique was applied. After the target vessel was opened, balloon dilation was used, and stents were implanted according to the indications after angiography. All stents were self-expanding NiTi alloy stents and were dilated after balloon. When the puncture site was seriously calcified, the arterial hemostat
(Beijing Puyi Shengii Technology Co., Ltd.) was used, and the other was Perclose ProGlide (Abbott vacuum).

\section{Drug administration}

Aspirin enteric coated tablets $(100 \mathrm{mg} / \mathrm{d})$ and clopidogrel bisulfate tablets $(75 \mathrm{mg} / \mathrm{d})$ were used for at least 3 days before operation. Aspirin enteric coated tablets and low molecular weight heparin (100 U/kg, Q12 h) were administered postoperatively. Five days later, aspirin enteric coated tablets and clopidogrel bisulfate tablets were administrated. Aspirin enteric coated tablets were maintained for a long time after 1 year. Patients with combined diseases were given corresponding drug treatment.

\section{Statistical analysis}

SPSS24.0 was used to calculate the patency rate, survival rate and limb salvage rate between the two groups. Kaplan Meier method was used to calculate the patency rate, survival rate and limb salvage rate. Log rank test was used for univariate analysis. The difference was considered statistically significant when $\mathrm{P}$ values were less than 0.05 .

\section{Results}

\section{General information}

The patients included 29 males and 10 females, aged from 60 to 82 years, with an average of $70.5 \pm 6.4$ years. The BMI of the patients was 21.4-34.6, with an average of $25.4 \pm 3.9$. The course of disease was 1-22 months, with an average of $10.3 \pm 5.2$ months. The length of hospital stay was 7-19 days, with an average of $12.5 \pm 4.0$ days. There were 39 patients with Fontaine IV, including 31 cases of Rutherford grade 5 and 8 cases of Rutherford grade 6 . There were 21 cases on the left side, 18 cases on the right side, 25 cases with ulcer and 14 cases with gangrene. The risk factors included smoking in 23 cases, hyperlipidemia in 26 cases, hypertension in 27 cases, diabetes mellitus in 17 cases and endstage renal disease in 12 cases.

31 cases received local anesthesia and 8 cases received general anesthesia. The length of occlusive lesion was $32.0 \pm 7.1 \mathrm{~cm}$, the operation time was $122.5 \pm 33.2 \mathrm{~min}$, and the dosage of contrast medium was $146.0 \pm 33.0 \mathrm{ml}$. According to the approach, 30 cases were reversely punctured, 9 cases were antegrade, 22 cases were true lumen opening, 16 cases were subintimal, 5 cases were reversely punctured, 7 cases were balloon dilatation, 32 cases were balloon dilatation+stent implantation. The average number of stents per patient was 1.7, hemostatic devices were 13 cases, and suture devices were 26 cases. The clinical and technical success rates were $100.0 \%$ (39/39). The average $\mathrm{ABI}$ was $0.89 \pm 0.13$ after operation, which was significantly higher than the value of preoperation $(0.17 \pm 0.12, \mathrm{P}<0.001)$. The perioperative complications included 2 cases of myocardial infarction, 1 case of ischemic stroke, and 1 case of pseudoaneurysm at puncture point. In stent restenosis rate was $43.6 \%$ (17/39). The patients were followed up for 24-1692 days (median 1246 days).

\section{Vascular patency rate and influencing factors}

The primary patency rates of 1 year, 2 years, and 3 years were $94.5 \%$, $75.1 \%$ and $55.6 \%$, respectively, with a median time of 1170 days. The assisted-primary patency rates of 1 year, 2 years, and 3 years were $94.5 \%, 80.6 \%$ and $63.6 \%$ respectively, and the median time was 1230 days. The secondary patency rates were $94.5 \%, 86.2 \%$ and $69.0 \%$, respectively. The median time was 1260 days (Figure 1). Univariate analysis showed that common femoral artery lesions involving the superficial and profunda femoral artery bifurcation, diabetes mellitus and end-stage renal disease were associated with primary patency rate, 


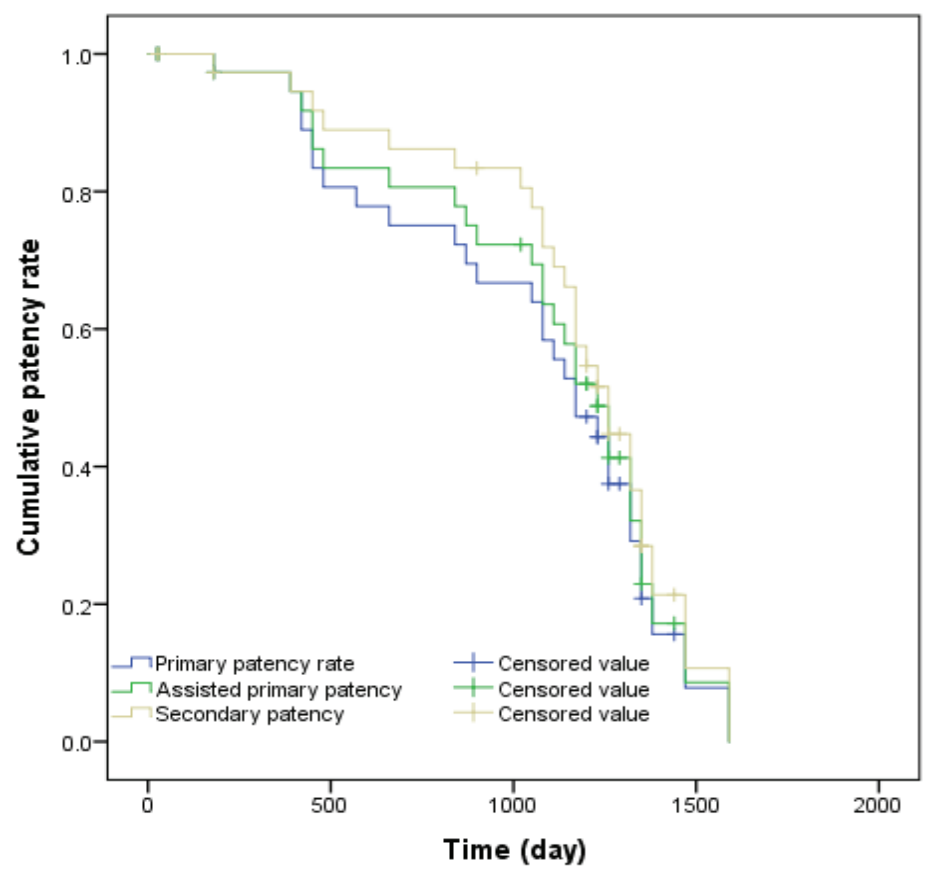

Figure 1: Vascular patency curve.

but age, gender, smoking, hypertension, lesion length, true lumen opening and stent implantation were not related to primary patency rate (Table 1).

\section{Overall survival rate and influencing factors}

The 1-year, 2-year and 3-year overall survival rates of 39 patients were $89.7 \%, 76.9 \%$ and $67.3 \%$, respectively. The median time was 1246 days (Figure 2). The causes of death included 4 cases of ischemic stroke, 1 case of hemorrhagic stroke, 4 cases of ischemic heart disease, 1 case of chronic obstructive pulmonary disease, 1 case of road traffic injury and 1 case of malignant tumor. Univariate analysis showed that end-stage renal disease, coronary heart disease and gangrene were associated with overall survival rate, but age, gender, smoking, body mass index, hypertension, diabetes mellitus, cerebrovascular disease and stent were not related to overall survival rate (Table 2).

\section{Limb salvage rate and influencing factors}

The total amputation rates of 39 cases were $71.8 \%, 64.1 \%$ and $53.5 \%$ in 1 year, 2 years and 3 years, respectively (Figure 3 ). By at the end of follow-up, 18 amputations were performed, including $28.0 \%$ (7/25) in ulcer group and $78.6 \%(11 / 14)$ in gangrene group, including 8 cases of large amputation and 10 cases of small amputation. Univariate analysis showed that age, diabetes, end-stage renal disease and gangrene were related to limb salvage rate, but age, gender, smoking, hypertension, common femoral artery involvement, lesion length and stent were not related to limb salvage rate (Table 3).

\section{Ulcer healing}

The ulcer area of 25 patients was $0.6-7.2 \mathrm{~cm}^{2}$, with an average of 3.4 $\pm 1.9 \mathrm{~cm}^{2}$. The ulcer healing rate was $88.0 \%(22 / 25)$. The healing time of 22 cases was $8-72$ days, with an average of $38.3 \pm 17.2$ days. The healing rate of ulcer was $32.0 \%$ in 30 days and $76.0 \%$ in 60 days. Amputation was performed in 2 cases and non-healing in 1 case (Figure 4).

\section{Discussion and Conclusion}

PAD is a chronic disease with high social health risk which is seriously neglected and underestimated. In this study, all patients were over 60 years old with TASC II D femoropopliteal artery disease ulcer gangrene, and there were many risk factors. The incidence of cardiovascular and cerebrovascular events was high in patients with PAD of lower limbs during perioperative period [12]. The incidence of cardiovascular and cerebrovascular events in this study was $7.7 \%$. The use of suture device at the puncture point after operation can make the patient walk around early and recover quickly. In this study, one case of pseudoaneurysm occurred after using suture device, which is related to the serious calcification of puncture point.

The patency rate of this study was higher than that of other related research [6], which was mainly related to the requirements of inflow and outflow tract grouping standards. It has been reported that in stent restenosis was a significant problem after endovascular treatment, with an incidence of $40 \%-50 \%$, and the most common occurrence is in the femoral popliteal artery [13]. Plaque rotation system as an effective treatment of in stent restenosis has achieved good clinical effect [14]. In this study, $43.6 \%$ patients had in stent restenosis occlusion. 4 cases were treated with this method and the clinical effect was satisfactory. The primary patency rate involving the common femoral artery is lower than that of the group of simple femoral popliteal artery. It may be located at the bifurcation of the femoral artery near the joint with the common femoral artery, which has a large range of motion and complex hemodynamics. It has been reported that open surgery can deal with the occlusive lesions of the common femoral artery and protect the deep femoral artery [15]. The low vascular patency rate in end-stage renal disease may be related to severe vascular calcification and less collateral formation. Diabetic patients with vascular endothelial cell damage, platelet aggregation and adhesion enhancement, blood hypercoagulability, etc., are also common clinical 


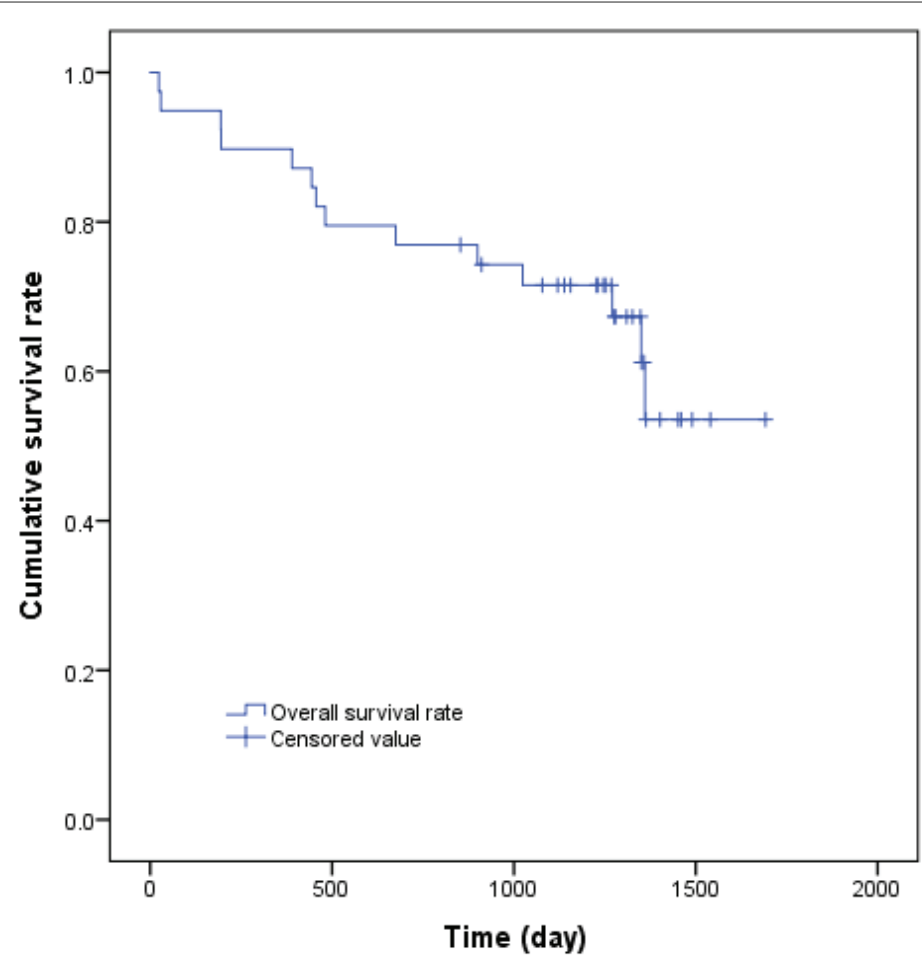

Figure 2: Overall survival curve.

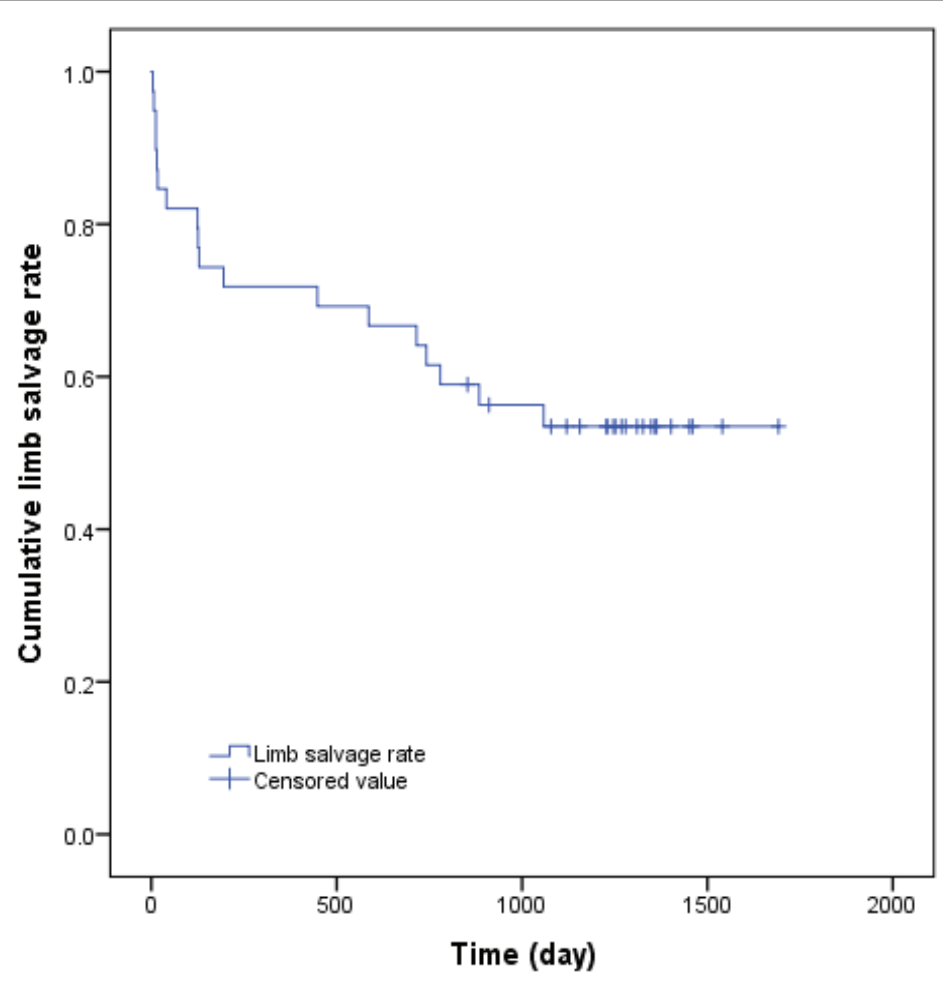

Figure 3: Limb salvage curve. 


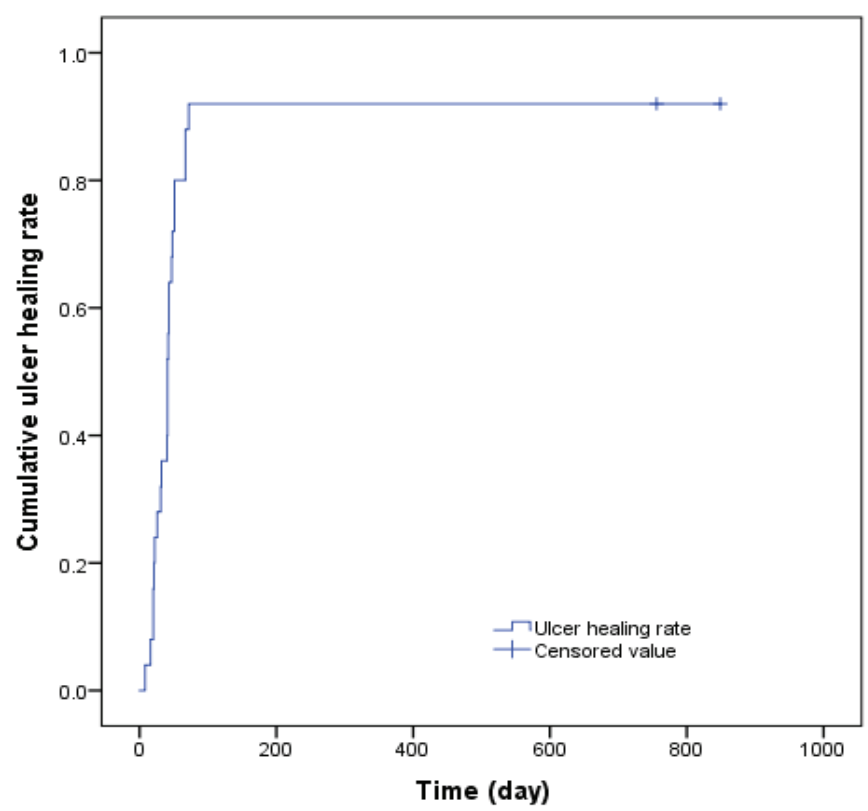

Figure 4: Ulcer healing curve.

Table 1: The factors affecting primary potency.

\begin{tabular}{|l|c|c|c|c|}
\hline & Cases & Mean \pm SD (Y/N) & $\boldsymbol{X}^{2}$ value & P value \\
\hline Age $(>70$ years) & $20 / 19$ & $930.1 \pm 98.8 / 1169.3 \pm 84.1$ & 2.981 & 0.084 \\
\hline Male & $29 / 10$ & $1042.7 \pm 86.5 / 1073.0 \pm 94.1$ & 0.040 & 0.841 \\
\hline Smoking & $23 / 16$ & $1003.2 \pm 93.7 / 1096.4 \pm 93.1$ & 0.232 & 0.630 \\
\hline Hypertension & $27 / 12$ & $1071.1 \pm 89.7 / 1014.4 \pm 99.5$ & 0.228 & 0.633 \\
\hline Diabetes & $17 / 22$ & $860.0 \pm 98.7 / 1219.0 \pm 81.1$ & 8.520 & 0.004 \\
\hline End-stage renal disease & $12 / 27$ & $722.7 \pm 126.1 / 1201.6 \pm 62.4$ & 9.636 & 0.002 \\
\hline Common femoral artery & $26 / 13$ & $840.0 \pm 103.3 / 1222.3 \pm 70.5$ & 7.509 & 0.006 \\
\hline Length of lesion>30 cm & $21 / 18$ & $1007.2 \pm 88.9 / 1104.6 \pm 103.6$ & 3.422 & 0.064 \\
\hline True cavity opening & $22 / 17$ & $1055.4 \pm 82.1 / 1038.0 \pm 109.6$ & 0.017 & 0.897 \\
\hline Vascular stent & $32 / 7$ & $1099.4 \pm 75.1 / 861.4 \pm 129.0$ & 2.406 & 0.121 \\
\hline
\end{tabular}

Table 2: The factors affecting overall survival rates.

\begin{tabular}{|c|c|c|c|c|}
\hline & Cases & Mean $\pm S D(Y / N)$ & $\chi^{2}$ value & $P$ value \\
\hline Age (>70 years) & $20 / 19$ & $1009.3 \pm 124.6 / 1454.5 \pm 109.0$ & 3.843 & 0.050 \\
\hline Male & $29 / 10$ & $1193.8 \pm 115.8 / 1348.6 \pm 90.1$ & 1.262 & 0.261 \\
\hline Smoking & $23 / 16$ & $1127.1 \pm 132.0 / 1349.5 \pm 83.3$ & 3.269 & 0.071 \\
\hline $\mathrm{BMI}>24.0$ & $21 / 18$ & $1136.8 \pm 132.6 / 1309.9 \pm 120.3$ & 3.422 & 0.064 \\
\hline Hypertension & $27 / 12$ & $1285.9 \pm 121.3 / 1173.8 \pm 112.1$ & 0.318 & 0.573 \\
\hline Diabetes & $17 / 22$ & $1061.6 \pm 121.7 / 1404.4 \pm 117.7$ & 3.047 & 0.081 \\
\hline End-stage renal disease & $12 / 27$ & $829.8 \pm 153.0 / 1442.7 \pm 95.0$ & 10.760 & 0.001 \\
\hline Coronary heart disease & $17 / 22$ & $780.7 \pm 133.4 / 1189.7 \pm 92.0$ & 5.085 & 0.024 \\
\hline Cerebrovascular disease & $6 / 31$ & $780.8 \pm 196.7 / 1037.5 \pm 92.0$ & 0.082 & 0.774 \\
\hline Vascular stent & $32 / 7$ & $1288.8 \pm 104.6 / 1018.5 \pm 138.7$ & 0.449 & 0.503 \\
\hline Gangrene & $14 / 25$ & $874.4 \pm 151.8 / 1448.8 \pm 92.0$ & 6.418 & 0.011 \\
\hline
\end{tabular}


Table 3: The factors affecting limb salvage rate.

\begin{tabular}{|c|c|c|c|c|}
\hline & Cases & Mean \pm SD $(Y / N)$ & $\chi^{2}$ value & P value \\
\hline Age (>70 years) & $20 / 19$ & $691.7 \pm 138.6 / 1344.7 \pm 139.2$ & 6.801 & 0.009 \\
\hline Male & $29 / 10$ & $1026.2 \pm 141.5 / 986.9 \pm 136.2$ & 0.004 & 0.947 \\
\hline Smoking & $23 / 16$ & $923.3 \pm 158.8 / 1109.8 \pm 127.2$ & 1.292 & 0.256 \\
\hline Hypertension & $27 / 12$ & $1129.8 \pm 137.2 / 806.5 \pm 176.9$ & 0.960 & 0.327 \\
\hline Diabetes & $17 / 22$ & $696.9 \pm 142.5 / 1262.9 \pm 140.9$ & 4.098 & 0.043 \\
\hline End-stage renal disease & $12 / 27$ & $521.5 \pm 153.6 / 1260.1 \pm 125.2$ & 7.106 & 0.008 \\
\hline Common femoral artery & $26 / 13$ & $763.3 \pm 128.5 / 1258.5 \pm 144.3$ & 3.347 & 0.068 \\
\hline Length of lesion $>30 \mathrm{~cm}$ & $21 / 18$ & $809.3 \pm 122.0 / 1188.4 \pm 169.8$ & 1.762 & 0.184 \\
\hline Vascular stent & $32 / 7$ & $1078.9 \pm 129.7 / 797.0 \pm 182.0$ & 0.188 & 0.665 \\
\hline Gangrene & $14 / 25$ & $532.9 \pm 138.4 / 1305.0 \pm 127.1$ & 10.458 & 0.001 \\
\hline
\end{tabular}

problems after endovascular treatment of postoperative restenosis [16].

The 5-year mortality rate of patients with severe limb ischemia can reach 50\%-70\% [17], and once vascular reconstruction fails, they will face $40 \%$ amputation rate and $20 \%$ mortality rate within 6 months [4]. The 3 -year survival rate was $67.3 \%$. The main cause of death was stroke $38.5 \%$. Gangrene and end-stage renal disease were the main influencing factors, which were related to the progress of the disease course [18]. The 1-year and 3-year limb salvage rates were $71.8 \%$ and $53.5 \%$ respectively, which were lower than those in the previous study [6]. The difference in the results was related to the different subjects included. PAD of lower limbs can cause diabetic foot ulcer and gangrene, which is the main reason of amputation in diabetic patients. Patients with end-stage renal disease have higher amputation rate and mortality rate after vascular reconstruction [19]. Gangrene is mainly tissue necrosis and secondary infection of spoilage bacteria, which has a higher risk of amputation than ulcer. Amputation after vascular reconstruction is the main treatment [20]. The amputation rate of gangrene patients in this study is $78.6 \%$, and the other 3 cases were successfully limb salvage by skin flap transplantation and other techniques. Most of the wounds healed well after vascular reconstruction. The ulcer healing rate was $88.0 \%$ after intracavitary treatment. There was one diabetic patient whose ulcer had not completely healed by the end of follow-up. Due to the small number of cases in this group, the risk factors affecting the ulcer healing rate were not statistically analyzed, and the relevant literature did not make a clear conclusion.

It has been reported that the target vessel occlusion of severe limb ischemia is based on thrombosis on the basis of atherosclerosis. Anticoagulation can improve the limb salvage rate [21]. Low molecular weight heparin was routinely used for anticoagulation. In addition, health education for patients during follow-up, such as smoking cessation and alcohol restriction, blood pressure control, cholesterol reduction and exewrcise therapy can also play an irreplaceable role in delaying the progress of PAD after endovascular treatment [22].

In conclusion, the mid-term follow-up effect of endovascular treatment for ulcerative gangrene patients with TASC II D is acceptable. However, there are still many uncertainties in the long-term follow-up effect compared with open surgery.

\section{Limitation}

Our study has important limitations. First, this is a retrospective observational study. Second, we were unable to account for the effectiveness of endovascular or surgical revascularization procedures since there was no comparison with surgical arm in this study. Last, outcomes are limited to small sample size on factors with effect patency, survival and salvage rate.

\section{Funding}

The study is funded by Medical Science Research Project of the key research and development program of Hebei Province, China (No: 20377732D).

\section{References}

1. Aronow WS (2012) Peripheral arterial disease of the lower extremities. Arch Med Sci 8: 375-388.

2. Kinlay S (2016) Management of Critical Limb Ischemia. Circ Cardiovasc Interv 9: 1946.

3. Stoner MC, Calligaro KD, Chaer RA, Dietzek AM, Farber A, et al. (2016) Reporting standards of the Society for Vascular Surgery for endovascular treatment of chronic lower extremity peripheral artery disease. J Vasc Surg 64: 1-21.

4. Norgren L, Hiatt WR, Dormandy JA, Nehler MR, Harris KA, et al. (2007) Inter-Society Consensus for the Management of Peripheral Arterial Disease (TASC II). Eur J Vasc Endovasc Surg 1: 1-75.

5. Biagioni RB, Brandão GD, Biagioni LC, Nasser F, Burihan MC, et al. (2019) Endovascular treatment of TransAtlantic Inter-Society Consensus II D femoropopliteal lesions in patients with critical limb ischemia. J Vasc Surg 69: 1510-1518.

6. Patel SD, Biasi L, Paraskevopoulos I, Silickas J, Lea T, et al. (2016) Comparison of angioplasty and bypass surgery for critical limb ischaemia in patients with infrapopliteal peripheral artery disease. Br J Surg 103: 1815-1822.

7. Bracale UM, Giribono AM, Spinelli D, Guercio LD, Pipitò N, et al. (2019) Long-term Results of Endovascular Treatment of TASC C and D Aortoiliac Occlusive Disease with Expanded Polytetrafluoroethylene Stent Graft. Ann Vasc Surg 56: 254-260.

8. Bosiers M, Setacci C, Donato GD, Torsello G, Silveira PG, et al. (2020) ZILVERPASS Study: ZILVER PTX Stent vs Bypass Surgery in Femoropopliteal Lesions. J Endovasc Ther 27: 287-295.

9. Brachial Index CA; Fowkes FGR, Murray GD, Butcher I, Heald CL (2008) Ankle brachial index combined with Framingham Risk Score to predict cardiovascular events and mortality: a meta-analysis. JAMA 300: 197-208. 
10. Liu P, Peng J, Zheng L, Lu H, Yu W, et al. (2018) Application of computed tomography venography in the diagnosis and severity assessment of iliac vein compression syndrome: A retrospective study. Medicine (Baltimore) 97: e12002.

11. Chinese Medical Association (2015) Guidelines for diagnosis and treatment of arteriosclerosis obliterans of lower extremities. Chinese J Med 95: 1883-1996.

12. Banerjee S, Sarode K, Mohammad A, Gigliotti O, Baig MS, et al. (2016) Femoropopliteal Artery Stent Thrombosis: Report from the Excellence in Peripheral Artery Disease Registry. Circ Cardiovasc Interv 9: 002730.

13. Bradbury AW (2009) Bypass versus angioplasty in severe ischaemia of the leg (BASIL) trial: what are its implications? Semin Vasc Surg 22: 267-274.

14. Loffroy R, Edriss N, Goyault G, Chabanier A, Pernes JM, et al. (2020) Percutaneous mechanical atherothrombectomy using the Rotarex ${ }^{\circledR}$ $S$ device in peripheral artery in-stent restenosis or occlusion: a French retrospective multicenter study on 128 patients. Quant Imaging Med Surg 10: 283-293.

15. An C, Fang H, Yang Y, Sun X, Liu Q, et al. (2019) Correlation between serum high mobility group protein $\mathrm{B} 1$ level and restenosis after stent implantation in patients with type 2 diabetes mellitus. Chinese J Diabetes 27: 429-433.

16. Ahanchi SS, Panneton JM, Stout CL (2013) A hybrid approach to recanalization of a chronic iliofemoral occlusion. J Vasc Surg 57: 230 233.
17. Caro J, Migliaccio-Walle K, Ishak KJ, Proskorovsky I (2005) The morbidity and mortality following a diagnosis of peripheral arterial disease: long-term follow-up of a large database. BMC Cardiovasc Disord 5: 14.

18. YuoT H, Chaer RA, Dillavou ED, Leers SA, Makaroun MS, et al. (2015) Patients started on hemodialysis with tunneled dialysis catheter have similar survival after arteriovenous fistula and arteriovenous graft creation. J Vasc Surg 62: 1590-1597.

19. You TH, Wallace JR, Fish L, Avgerinos ED, Leers SA, et al. (2019) Editor's Choice - Comparison of Outcomes After Open Surgical and Endovascular Lower Extremity Revascularisation Among End Stage Renal Disease Patients on Dialysis. Eur J Vasc Endovasc Surg 57: 248257.

20. Zhan LX, Branco BC, Armstrong DG, Mills Sr JL (2015) The Society for Vascular Surgery lower extremity threatened limb classification system based on Wound, Ischemia, and foot Infection (WIfI) correlates with risk of major amputation and time to wound healing. J Vasc Surg 61: 939-944.

21. Narula N, Dannenberg AJ, Olin JW, Bhatt DL, Johnson KW, et al. (2018) Pathology of Peripheral Artery Disease in Patients With Critical Limb Ischemia. J Am Coll Cardiol 72: 2152-2163.

22. Bevan GH, Solaru KTW (2020) Evidence-Based Medical Management of Peripheral Artery Disease. Arterioscler Thromb Vasc Biol 40: 541553 